PROCEEDINGS OF THE AMERICAN MATHEMATICAL SOCIETY

Volume 124, Number 10, October 1996

\title{
ON THE FAILURE OF CLOSE-TO-NORMAL STRUCTURE TYPE CONDITIONS AND PATHOLOGICAL KANNAN MAPS
}

\author{
MICHAEL A. SMYTH
}

(Communicated by Palle E. T. Jorgensen)

\begin{abstract}
We consider the failure of close-to-normal structure type conditions and show that a Banach space can be renormed to fail close-to-weak normal structure exactly when it contains a norm inseparable weakly compact subset. Included is an example of a particularly pathological fixed point free Kannan map.
\end{abstract}

1.

Throughout $X$ will denote a real Banach space. We recall that $X$ is said to have (weak) normal structure if whenever $C$ is a closed (weak compact) bounded convex subset of $X$ with $\operatorname{diam} C>0$, then $\operatorname{rad} C<\operatorname{diam} C$ where

$$
\operatorname{diam} C:=\sup \{\|x-y\|: x, y \in C\} \quad \text { and } \operatorname{rad} C:=\inf _{x \in C} \sup \{\|x-y\|: y \in C\}
$$

are the diameter and radius of the set $C$. We will denote normal structure and weak normal structure by, respectively, ns and w-ns. If $X$ is a dual space, it has weak star normal structure ( $\mathrm{w}^{*}$-ns) if we require the set $C$ of the above definition to be weak star compact. A Banach space $X$ has uniform normal structure if

$$
\begin{aligned}
\sup & \left\{\frac{\operatorname{rad} C}{\operatorname{diam} C}: C \text { nonempty nonsingleton closed bounded convex subset of } X\right\} \\
& <1 .
\end{aligned}
$$

The above normal structure type conditions have been useful in the fixed point theory of nonexpansive maps (see [2] for example). A Banach space $X$ has the fixed point property (weak fixed point property) if, given a nonempty closed (weak compact) bounded convex subset $C$ of $X$ that is self-mapped by a nonexpansive map $T$, then $T$ has a fixed point in $C$ (recall that $T: C \rightarrow C$ is nonexpansive if $\|T x-T y\| \leq\|x-y\|$ for all $x, y \in C)$. We abbreviate the two properties to fpp and w-fpp. If $X$ is a dual space, then it has the weak star fixed point property ( $\mathrm{w}^{*}$-fpp) if we require the set $C$ of the above definition to be weak star compact. It is known that w-ns implies the $\mathrm{w}$-fpp and that $\mathrm{w}^{*}$-ns implies the $\mathrm{w}^{*}$-fpp.

A Banach space $X$ is said to have close-to-normal structure (close-to-ns) if, given a nonempty nonsingleton closed bounded convex subset $C$ of $X$, there exists

Received by the editors February 23, 1995.

1991 Mathematics Subject Classification. Primary 46B20, 47H09.

(C)1996 American Mathematical Society 
$x \in C$ so that $\|x-y\|<\operatorname{diam} C$ for any $y \in C$. We can also define close-toweak normal structure (close-to-w-ns) and (if $X$ is a dual space) close-to-weak star normal structure (close-to-w*-ns) by replacing "closed bounded" in the above by "weak compact" or "weak star compact."

A self-map $T$ of a subset $C$ of a Banach space $X$ is a Kannan map if

$$
\|T x-T y\| \leq \frac{1}{2}(\|T x-x\|+\|T y-y\|)
$$

for all $x, y \in C$. We will say that $X$ has the weak fixed point property (w-fpp) for Kannan maps if, given a nonempty convex weak compact subset $C$ of $X$ that is self-mapped by a Kannan map $T$, then $T$ has a fixed point. The $\mathrm{w}^{*}$-fpp for Kannan maps can be defined when $X$ is a dual space by replacing "weak compact" with "weak star compact" in the above.

Wong [12] showed that $X$ has the w-fpp for Kannan maps if and only if it has close-to-w-ns. The results stated in [6] give that close-to-w*-ns is equivalent to the $\mathrm{w}^{*}$-fpp for Kannan maps. It is shown in [11] that spaces which are separable or strictly convex have close-to-ns and that the KK property implies close-to-w-ns. This is seen by the use of a convex series. Suppose that $C$ is a closed bounded nonempty and convex subset of a Banach space and that $A$ is a countable subset of $C$. Then $\overline{\operatorname{co}} A$ is a separable subset of $C$. Let $\left\{x_{n}\right\}_{n=1}^{\infty}$ be a countable dense subset of $\overline{c o} A$. Consider the point $x=\Sigma x_{n} / 2^{n}$. Since $C$ is a closed bounded convex subset of a Banach space, $x$ exists and is in $C$. Now it is easily seen that if $y \in C$ (so $\left\|x_{n}-y\right\| \leq \operatorname{diam} C$ for all $\left.n\right)$ and $\|x-y\|=\operatorname{diam} C$, then $\left\|x_{n}-y\right\|=\operatorname{diam} C$ for all $n$. But this also gives that $\operatorname{dist}(y, \overline{\operatorname{co}} A)=\operatorname{diam} C$. This procedure can be used to establish all of the results from [11] that were stated above.

In Section 2 we give examples of spaces that fail the close-to-normal structure conditions defined above, covering what appears to be known so far.

In Section 3 we concern ourselves with the equivalence stated in the abstract and give an example of a fixed point free idempotent Kannan map.

\section{2.}

Perhaps the simplest example of a set violating close-to-w-ns (and thus closeto-ns) is in $c_{0}(\Gamma)$ for an uncountable $\Gamma$. Indeed, if the $x_{i}$ are the usual unit basis elements, then $C:=\overline{\mathrm{co}}\left\{x_{i}\right\}_{i \in \Gamma}$ is weak compact, convex, and, for any $x \in c_{0}(\Gamma)$ and $i \in \Gamma,\left\|x_{i}-x\right\| \geq 1$ if $i \notin \operatorname{supp} x$. Of course $\operatorname{diam} C=1$.

It is not hard to give nonconstructive subsets of $l_{\infty}$ violating close-to-ns, as in the following example.

Example 1. Let $\mathcal{U}$ be a free ultrafilter on $\mathbb{N}$. Put

$$
A:=\left\{x \in l_{\infty}: x(n) \in\{0,1\} \text { for } n \in \mathbb{N} \text { and }\{n: x(n)=0\} \in \mathcal{U}\right\}
$$

and

$$
C:=\overline{\mathrm{co}} A \text {. }
$$

Clearly $\operatorname{diam} C=1$. We show that for any $y \in C$ there exists $x \in A$ so that $\|y-x\|=1$. Indeed, suppose that $y \in C$ and $m \in \mathbb{N}$. Define

$$
B_{m}:=\{n \in \mathbb{N}: y(n) \leq 1 / m\} .
$$

Note that if $z \in \operatorname{co} A, z=\lambda_{1} x_{1}+\lambda_{2} x_{2}+\cdots+\lambda_{p} x_{p}$, say, with $x_{i} \in A, \sum \lambda_{i}=$ $1, \lambda_{i} \geq 0$, then there exists $U \in \mathcal{U}$ so that $z(n)=0$ for all $n \in U$. This will imply that the complement of $B_{m}$ is not in $\mathcal{U}$, giving $B_{m} \in \mathcal{U}$. 
It follows that there exists an infinite subsequence $\left(n_{m}\right)_{m \in \mathbb{N}}$ of $\mathbb{N}$ so that $y\left(n_{m}\right) \leq$ $1 / m$ for all $m \in \mathbb{N}$. But every infinite subset of $\mathbb{N}$ contains an infinite subset that is not in $\mathcal{U}$. Thus, we can extract a further sequence $\left(n_{m_{i}}\right)_{i \in \mathbb{N}}$ so that $y\left(n_{m_{i}}\right) \rightarrow_{i} 0$ and $M:=\left\{n_{m_{i}}\right\}_{i \in \mathbb{N}} \notin \mathcal{U}$. Then $\chi_{M} \in A$ and $\left\|y-\chi_{M}\right\|=1$, giving the result.

In [6] it is shown that $l_{\infty}$ fails close-to-w*-ns. Indeed their result is that if $(\Omega, \Sigma, \mu)$ is a sigma finite measure space (so that the dual of $L_{1}(\Omega, \Sigma, \mu)$ is $\left.L_{\infty}(\Omega, \Sigma, \mu)\right)$, then $L_{\infty}(\Omega, \Sigma, \mu)$ fails close-to-w*-ns if it is inseparable. Of course, $l_{\infty}$ has close-to-w-ns by the result of Wong given earlier, since all of its weak compacts are (norm) separable. In [4] the above result from [6] is used to show that if $X$ is an infinite dimensional Hilbert space, then $B(X)$, the space of bounded linear operators on $X$, fails close-to-w*-ns. It is also shown that the space of compact operators on $X$ has close-to-w-ns if and only if $X$ is inseparable but that the space of trace class operators always has close-to- $\mathrm{w}^{*}$-ns. It was subsequently shown in [5] that the trace class has $\mathrm{w}^{*}$-ns.

Suppose that $\Omega$ is a compact Hausdorff space. Then $C(\Omega)^{*}$, the space of continuous real valued functions on $\Omega$ with the supremum norm, fails close-to-w*-ns exactly when $\Omega$ is uncountable (that is, when $C(\Omega)^{*}$ is inseparable). To verify this, we first identify $C(\Omega)^{*}$ with $M(\Omega)$, the space of radon measures on $\Omega$ with total variation norm, the actions on $C(\Omega)$ being integration. If $\Omega$ is countable, to show that $C(\Omega)^{*}$ has close-to- $\mathrm{w}^{*}$-ns we can assume that $\Omega$ is infinite. Then $M(\Omega) \equiv l_{1}$, a separable space which thus has close-to-w*-ns from above. If $\Omega$ is uncountable, consider

$$
C:=\{\mu \in M(\Omega):\|\mu\| \leq 1, \mu(\Omega)=1\} .
$$

$C$ is the intersection of the $\mathrm{w}^{*}$ compact unit ball and a $\mathrm{w}^{*}$ closed hyperplane and so is thus convex and $\mathrm{w}^{*}$ compact. Clearly $\operatorname{diam} C=2$. If $\mu \in C$, then $\{x \in \Omega: \mu(\{x\})=0\} \neq \varnothing$ since $\Omega$ is uncountable. Now if $\delta_{x}$ denotes the dirac measure at $x$, then $\left\|\mu-\delta_{x}\right\|=2$. Thus $C(\Omega)^{*}$ fails close-to-w*-ns.

We make a slight digression here on the failure of $\mathrm{w}^{*}$-ns and the $\mathrm{w}^{*}$-fpp. Suppose that $K$ is a locally compact Hausdorff space with $C_{0}(K)$ denoting the space of real valued continuous functions on $K$ vanishing at infinity. In [4] it was asked when does $C_{0}(K)^{*}$ fail w*-ns? We note here that $C_{0}(K)^{*}$ fails w*-ns (and also the w*fpp) exactly when $K$ is nondiscrete. To verify this, we identify $C_{0}(K)^{*}$ with $M(K)$, the space of radon measures on $K$. As in [9], if $\Omega$ is a compact subset of $K$, then $C(\Omega)^{*}$ is isometrically isomorphic to a $\mathrm{w}^{*}$ closed subspace of $C_{0}(K)^{*}$ via a w* homeomorphism (namely, the map which extends a measure to be identically zero outside $\Omega$ ). Now if $K$ is nondiscrete, then, by the local compactness, it contains an infinite compact subset $\Omega$. But $C(\Omega)^{*}$ fails the $\mathrm{w}^{*}$-fpp by a result from [9], and thus so does $C_{0}(K)^{*}$. Otherwise, if $K$ has the discrete topology, then $C_{0}(K)^{*} \equiv c_{0}(K)^{*}$, well known to have $\mathrm{w}^{*}$-ns (and thus the $\mathrm{w}^{*}$-fpp).

We now give a general method for producing spaces which fail close-to-ns before considering renorming results. Suppose that the Banach space $X$ fails uniform normal structure. That is, for every $m \in \mathbb{N}$ there exists a closed convex subset $C_{m}$ of $X$ so that $\operatorname{diam} C_{m}=1$ and $\operatorname{rad} C_{m} \geq 1-1 / m$. We can also assume that $0 \in C_{m}$ for all $m$. Put $Y:=l_{\infty}(X)$ and

$$
C:=\prod_{m} C_{m}=\left\{\left(x_{m}\right) \in l_{\infty}(X): x_{m} \in C_{m} \text { for all } m\right\} .
$$


Clearly $C$ is closed, convex and $\operatorname{diam} C=1$. Now suppose that $\left(x_{m}\right) \in C$. For any $m$ there exists $y_{m} \in C_{m}$ so that $\left\|x_{m}-y_{m}\right\|>1-2 / m$. Then $\left\|\left(x_{m}\right)-\left(y_{m}\right)\right\|=1$, showing that $Y$ fails close-to-ns.

The above example can be further refined using ultrapowers. For material on ultrapowers see, for example, [3], [7] or [2]. Suppose $X$ is as above and $\mathcal{U}$ is a free ultrafilter on $\mathbb{N}$. Let $\tilde{X}$ be the associated ultrapower of $X$, so $\tilde{X}=l_{\infty}(X) / \mathcal{N}(\mathcal{U})$, where

$$
\mathcal{N}(\mathcal{U}):=\left\{\left(x_{n}\right) \in l_{\infty}(X): \lim _{\mathcal{U}}\left\|x_{n}\right\|=0\right\}
$$

Now define

$$
\widetilde{C}:=\left(\prod_{m} C_{m}\right)_{\mathcal{U}}=\left\{\left(x_{m}\right)_{\mathcal{U}} \in \widetilde{X}: x_{m} \in C_{m} \text { for all } m\right\} .
$$

Then $\widetilde{C}$ is closed, convex and bounded with $\operatorname{diam} \widetilde{C}=1$. Also if $\left(x_{m}\right) \mathcal{U} \in \widetilde{C}$ and we choose the $y_{m}$ as above, then $\left\|x_{m}-y_{m}\right\| \rightarrow 1$, so $\left\|\left(x_{m}\right)_{\mathcal{U}}-\left(y_{m}\right) \mathcal{U}\right\|_{\mathcal{U}}=$ $\lim _{\mathcal{U}}\left\|x_{m}-y_{m}\right\|=1$, showing that $\tilde{X}$ fails close-to-ns.

Of course if $X$ was originally superreflexive (so every ultrapower of $X$ is also superreflexive), then $\widetilde{X}$ is a superreflexive space that fails close-to-ns. We note that superreflexive spaces that fail uniform normal structure are easily given using a result from [1]: Every infinite-dimensional Banach space can be renormed to fail normal structure. More examples of superreflexive spaces failing close-to-ns are given below, where we are concerned with renorming spaces to fail close-to-ns or close-to-w-ns. The pathological sets so obtained will be similar to the example in $c_{0}(\Gamma)$ given above.

We start by recalling that a class of pairs $\left(x_{i}, x_{i}^{*}\right)_{i \in I}$, where $x_{i} \in X$ and $x_{i}^{*} \in X^{*}$, is called a biorthogonal system if $x_{i}^{*}\left(x_{j}\right)=\delta_{i}^{j}$ for any $i, j \in I$. The following proposition uses an adaptation of the technique for renorming to fail normal structure used in [1].

Proposition 2. Suppose that $X$ admits an uncountable biorthogonal system. Then $X$ can be renormed to fail close-to-ns.

Proof. Suppose $\left(x_{i}, x_{i}^{*}\right)_{i \in I}$ is the uncountable system. We can assume that the set $\left\{x_{i}\right\}_{i \in I}$ is bounded. Now, since $I$ is uncountable, there exists $N \in \mathbb{N}$ and an uncountable subset $J$ of $I$ so that $\left\|x_{i}^{*}\right\| \leq N$ for all $i \in J$. For simplicity we will assume that $I=J$. Now for $x \in X$ define

$$
\|x\|^{\prime}:=\max \left\{\frac{\|x\|}{\operatorname{diam}\left\{x_{i}\right\}_{i \in I}}, \sup _{i \in I}\left|x_{i}^{*}(x)\right|\right\} .
$$

Obviously $\left(X,\|\|^{\prime}\right)$ is isomorphic to $X$. Put $C:=\overline{\operatorname{co}}\left\{x_{i}\right\}_{i \in I}$. Clearly $\operatorname{diam} C=1$. Now suppose that $x \in \overline{\operatorname{span}}\left\{x_{i}\right\}_{i \in I}$. Then there exists a countable subset $A$ of $I$ so that $x \in \overline{\operatorname{span}}\left\{x_{i}\right\}_{i \in A}$. Thus, if we choose $j \in I \backslash A$, then $\left\|x_{j}-x\right\|^{\prime} \geq\left|x_{j}^{*}\left(x_{j}-x\right)\right|=1$. This obviously implies that $C$ violates close-to-ns.

If $X=l_{2}(I), I$ uncountable, with the $x_{i}$ the usual unit basis elements and $x_{i}^{*}$ the coordinate functionals, then, in the above,

$$
\|x\|^{\prime}=\max \left\{\frac{\|x\|_{2}}{2^{1 / 2}},\|x\|_{\infty}\right\},
$$


giving $Y:=\left(X,\|\|^{\prime}\right)$ isometric to an example used in [10], the norms differing by a multiplication by $2^{1 / 2}$. As in [10], the set $\left\{x \in Y: x(i) \geq 0\right.$ for all $i$ and $\left.\|x\|_{2} \leq 1\right\}$ can be used instead of $\overline{\mathrm{CO}}\left\{x_{i}\right\}$ to show failure of close-to-ns.

Also, note that if $X=c_{0}(I),\left(x_{i}, x_{i}^{*}\right)_{i \in I}$ the usual biorthogonal system, then \|\|$^{\prime}=\|\|_{\infty}$.

Not every inseparable Banach space has an uncountable biorthogonal system (see pg. 861 of [8]). It is unknown whether every inseparable Banach space can be renormed to fail close-to-ns. For the analogous close-to-w-ns problem the answer is given in the next section.

3.

First we recall some further material. A set $\left\{x_{i}\right\}_{i \in I}$ is said to be an M-basis of a Banach space $X$ if $\overline{\operatorname{span}}\left\{x_{i}\right\}_{i \in I}=X$ and there exists a total family $\left\{x_{i}^{*}\right\}_{i \in I} \subseteq X^{*}$ so that $\left(x_{i}, x_{i}^{*}\right)_{i \in I}$ is a biorthogonal system. Let $\delta(X)$ denote the density character of $X$. That is,

$$
\delta(X)=\min \{k: X \text { has a dense subset of cardinality } \leq k\} .
$$

In general, if $\left(x_{i}, x_{i}^{*}\right)_{i \in I}$ is a biorthogonal system, then $|I| \leq \delta(X)$ (see pg. 673 of [8]), and if $\left\{x_{i}\right\}_{i \in I}$ is an M-basis of $X$, then clearly $|I|=\delta(X)$ (assuming $X$ is infinite dimensional).

Theorem 3. A Banach space $X$ can be renormed to fail close-to-w-ns if and only if it contains a (norm) inseparable weak compact subset.

Proof. The forward implication is clear since any (norm) isomorphism is a weak homeomorphism.

For the reverse implication suppose that $X$ contains an inseparable weak compact set $C$. Then $Y:=\overline{\operatorname{span}} C$ is an inseparable weakly compactly generated subspace of $X$. By a well-known result, $Y$ can then be generated by a weak compact balanced convex set $K$.

Theorem 20.5(a) on pg. 693 of [8] will now give that $Y$ has an M-basis $\left\{x_{i}\right\}_{i \in I}$ with $x_{i} \in K$ for all $i$.

Since $\delta(Y)=|I|, I$ is uncountable. Since $K$ is weak compact, $\left\{x_{i}\right\}_{i \in I}$ is bounded. We can also extend the functionals associated with the M-basis to all of $X$ by Hahn-Banach and use the method of proof of the above proposition to produce an uncountable subset $J$ of $I$ and a renorming of $X$ so that the weak compact set $\overline{\mathrm{co}}\left\{x_{i}\right\}_{i \in J}$ violates close-to-w-ns.

The following corollary is now immediate.

Corollary 4. If $X$ is an inseparable weakly compactly generated space, then it can be renormed to fail close-to-w-ns.

A self-map $T$ of a nonempty set $C$ is periodic if there exists $n \in \mathbb{N}$ so that $T^{n}=I$. If $C$ is a nonempty subset of a Banach space $X$ and $T: C \rightarrow C$ is nonexpansive, then $T$ is said to be rotative if there exist $n \in \mathbb{N}$ and $a<n, a \in \mathbb{R}$, so that

$$
\left\|x-T^{n} x\right\| \leq a\|x-T x\| \text { for all } x \in C .
$$

Clearly a periodic nonexpansive map is rotative. Using the fixed point result concerning rotative maps that is given on pg. 177 of [2], we have that if $C$ is a nonempty closed convex subset of $X$, then any periodic nonexpansive self-map of $C$ has a fixed point. However, this is not true for periodic Kannan mappings. 
Proposition 5. Suppose that $X$ admits a biorthogonal system $\left(x_{i}, x_{i}^{*}\right)_{i \in I}$ with $|I|=$ $2^{\aleph_{0}}$. Then $X$ can be renormed so that the resulting space contains a nonempty bounded closed convex subset $C$ on which a fixed point free self-mapping Kannan map $T$ is defined satisfying $T^{2}=I$.

Proof. Since the cofinality of $2^{\aleph_{0}}$ is uncountable, there exists a subset $J$ of $I$ so that $|J|=2^{\aleph_{0}}$ and $N \in \mathbb{N}$ so that $\left\|x_{i}\right\| \leq N$ and $\left\|x_{i}^{*}\right\| \leq N$ for all $i \in J$.

We renorm $X$ as in the proof of Proposition 2, with

$$
\|x\|^{\prime}=\max \left\{\frac{\|x\|}{\operatorname{diam}\left\{x_{i}\right\}_{i \in J}}, \sup _{i \in J}\left|x_{i}^{*}(x)\right|\right\} .
$$

With $C:=\overline{\operatorname{co}}\left\{x_{i}\right\}_{i \in J}$ we note that the proof of Proposition 2 gives that if $x \in C$, then $\left|\left\{i \in J:\left\|x_{i}-x\right\|=1\right\}\right|=2^{\aleph_{0}}$. Also, since $\left(2^{\aleph_{0}}\right)^{\aleph_{0}}=2^{\aleph_{0}}$, it follows that $|C|=2^{\aleph_{0}}$. Write $C=\left\{y_{i}\right\}_{i<2^{\aleph_{0}}}$. Define $T: C \rightarrow C$ recursively as follows. Suppose that $T$ has been defined on $\left\{y_{i}\right\}_{i<\alpha}$ for some ordinal $\alpha<2^{\aleph_{0}}$.

Case 1. There exists $j<\alpha$ so that $T y_{j}=y_{\alpha}$. Then define $T y_{\alpha}:=y_{j}$.

Case 2. Otherwise. Put $F:=\left\{y_{i}\right\}_{i<\alpha} \cup\left\{T y_{i}\right\}_{i<\alpha}$. Then $|F|<2^{\aleph_{0}}$. From above, there exists $z \in C \backslash F$ so that $\left\|y_{\alpha}-z\right\|=1$. Now define $T y_{\alpha}:=z$.

Note that in the above procedure the $j$ in Case 1 is unique. Also $\|T x-x\|=1$ for all $x \in C$ and $\operatorname{diam} C=1$, so that $T$ is a fixed point free Kannan self-map of $C$. Finally, $T^{2}=I$. Indeed, suppose that $\alpha<2^{\aleph_{0}}$. First, suppose $T y_{\alpha}=y_{j}$ for some $j<\alpha$. Then $T y_{j}=y_{\alpha}$ from the definition of $T$, so $T^{2} y_{\alpha}=y_{\alpha}$. Otherwise, $T y_{\alpha}=y_{j}$ for some $j>\alpha$. Then, again from the definition of $T, T\left(y_{j}\right)=y_{\alpha}$ also giving $T^{2} y_{\alpha}=y_{\alpha}$.

By combining the methods of proof of Theorem 3 and Proposition 5, we obtain the following.

Corollary 6. Suppose $X$ contains a weak compact subset $K$ with $\delta(K) \geq 2^{\aleph_{0}}$. Then $X$ can be renormed so that the resulting space contains a nonempty weak compact convex subset $D$ on which a self-mapping fixed point free Kannan map is defined satisfying $T^{2}=I$.

\section{REFERENCES}

1. D. van Dulst, Equivalent norms and the fixed point property for nonexpansive mappings, J. London Math. Soc. 25 (1982), 139-144. MR 83e:47040

2. K. Goebel and W. A. Kirk, Topics in metric fixed point theory, Cambridge University Press, 1990. MR 92c: 47070

3. S. Heinrich, Ultraproducts in Banach space theory, J. Reine Angew. Mat. 313 (1980), 72-104. MR 82b: 46013

4. A. Lau and P. Mah, Quasi-normal structures for certain spaces of operators on a Hilbert space, Pacific J. Math. 121 (1986), 109-118. MR 87f:47065

5. C. Lennard, $\mathcal{C}_{1}$ is uniformly Kadec-Klee, Proc. Amer. Math. Soc. 109 (1990), 71-77. MR 90h: 46029

6. D. Roux and C. Zanco, Kannan maps in normed spaces, Rend. Sci. Fis. Mat. Nat. 65 (1978), 252-258. MR 81i:47062

7. B. Sims, Ultra-techniques in Banach space theory, Queen's Papers in Pure and Applied Mathematics, Vol. 60, 1982. MR 86h:46032

8. I. Singer, Bases in Banach spaces. II, Springer-Verlag, Berlin, Heidelberg, and New York, 1981. MR 82k: 46024

9. M. Smyth, Remarks on the weak star fixed point property in the dual of $C(\Omega)$, J. Math. Anal. Appl. 195 (1995), 294-306. CMP 96:01

10. K.-K. Tan, A note on asymptotic normal structure and close-to-normal structure, Canad. Math. Bull. 25 (1982), 339-343. MR 83i:46022 
11. C. Wong, Close-to-normal structure and its applications, J. Functional Anal. 16 (1974), 353358. MR 50:950

12. _ On Kannan maps, Proc. Amer. Math. Soc. 47 (1975), 105-111. MR 50:10929

Department of Mathematics, University of Auckland, Private Bag 92019, Auckland, New ZEALAND

Current address: 1 Frost Rd., Mt. Roskill, Auckland, New Zealand 